\title{
Swelling problems with two moving boundaries.
}

\author{
J. F. Caunce $^{1} \quad$ S. I. Barry ${ }^{2}$
}

(Received July 7, 2006; revised April 10, 2007)

\begin{abstract}
The swelling of grease and whole grains are modelled by a nonlinear diffusion equation with two moving boundaries (a Stefan problem). In Cartesian coordinates interesting analytical solutions exist for some simplified cases, but in general the solution must be found numerically. In cylindrical coordinates only numerical solutions are possible and these need the Cartesian results. This article develops models of the swelling material, illustrates some of the analytic solutions, and demonstrates the numerical methods used to solve the problem in Cartesian and cylindrical coordinates.
\end{abstract}

\section{Contents}

\section{Introduction}

See http://anziamj.austms.org.au/ojs/index.php/ANZIAMJ/article/view/41 for this article, (c) Austral. Mathematical Soc. 2007. Published May 8, 2007. ISSN $1446-8735$ 
2 Transformed equations

C123

3 Numerical Method

C124

4 Results

C127

5 Conclusion

C132

References

C132

\section{Introduction}

A Stefan problem $[6,8]$ with two moving boundaries is well posed by the non-dimensional equations

$$
\begin{aligned}
\frac{\partial u}{\partial t} & =\nabla(D(u) \nabla u), \quad x \in[S(t), R(t)], \\
\frac{d R}{d t} & =\left.a \frac{\partial u}{\partial r}\right|_{x=R}, \\
\frac{d S}{d t} & =-\left.b \frac{\partial u}{\partial r}\right|_{x=S},
\end{aligned}
$$

where $u(r, t)$ is a function of distance, $r$, and time, $t$, with initial condition $R(0)=S(0)$, and boundary conditions $u(S, t)=0$ and $u(R, t)=1$. The diffusivity term $D(u)$ may be nonlinear and is often written as $D=\exp (m u)$ for a constant $m$. No initial condition for $u(r, t)$ is needed since at $t=0$ the solution domain is the singular region, $r \in[R(0), R(0)]$. The parameters $a$ and $b$ are constants that relate to the physical properties of the material. Figure 1 illustrates the problem schematically.

This type of equation occurs in the modelling of swelling materials such as whole grains $[7,9]$. Our primary motivation is to model the swelling of a 


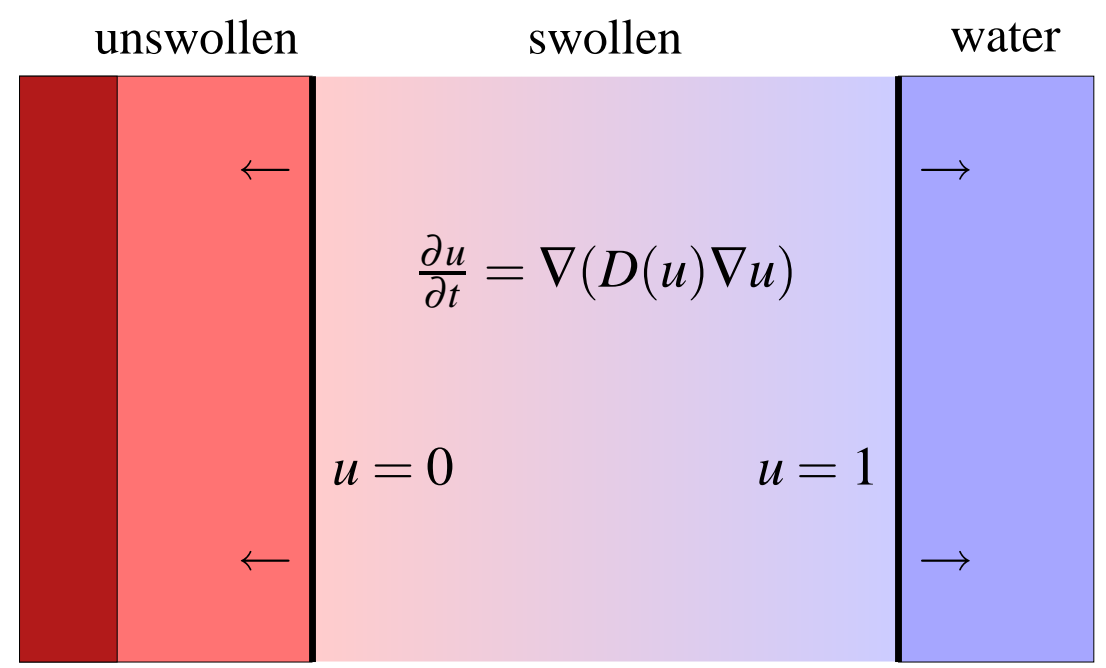

wool

$$
\begin{aligned}
x & =S(t) & x & =R(t) \\
S^{\prime}(t) & =-b \frac{\partial u}{\partial r}(S) & R^{\prime}(t) & =a \frac{\partial u}{\partial r}(R)
\end{aligned}
$$

Figure 1: Schematic diagram of a Stefan problem with two boundaries, $r=R(t), r=S(t)$, moving in opposite directions. Diffusion occurs between the boundaries, while the movement of the boundaries are governed by rate equations. 
grease layer on a wool fibre during the wool scouring process $[1,2,3,4,5]$ since a better understanding of the time for grease to swell could be used to improve scouring efficiency.

The governing equations are derived by Barry \& Caunce [2], as well as in slightly different form by Davey et al. [7, 9], for water diffusing into a polymer. The dependent variable in this case would be $\phi$, the moisture volume fraction, $\phi \in[0,1]$ with $\phi=1$ for water, and $\phi=0$ representing no water in the unswollen polymer. At the edges of the domain, $r=S(t)$ and $r=R(t)$, the volume fractions are $\phi=\phi_{0}$ and $\phi_{1}$ representing physical swollen and unswollen limits for the material. The diffusion equation governs movement of the moisture, with the rate equations, $R^{\prime}(t)$ and $S^{\prime}(t)$, derived by integration across the boundary $[2,7]$. To obtain the equations $(1-3)$ variables are scaled with respect to $\phi_{1}$, a length scale $x_{0}$ and a time scale $t_{0}=x_{0}^{2} / D^{*}\left(\phi_{1}\right)$. This scaling gives two constants:

$$
a=\frac{\phi_{1}-\phi_{0}}{1-\phi_{1}}, \quad b=\frac{D^{*}\left(\phi_{0}\right)}{D^{*}\left(\phi_{1}\right)} \frac{\phi_{1}-\phi_{0}}{\phi_{0}} .
$$

with $D^{*}$ being the diffusivity in dimensional quantities.

Most previous work on Stefan problems studied cases where one boundary is fixed or infinite and one boundary moves, such as occurs in many freezing or melting problems. The literature of these cases is well documented by Crank and Hill [6, 8].

The results presented here build on the work of Davey et al. [7, 9] who considered pseudo steady-state solutions, and Barry \& Caunce [2] who looked at analytic and numerical solutions in Cartesian geometries. Some of the detailed derivations will be left to these earlier works, while the major results are reproduced for completeness. The aim of our work is to extend our earlier results [2] to cylindrical coordinates and to explore numerical solutions for the more realistic case of nonlinear diffusivity, $D(u)=\exp (m u)$. We also explore solutions when the left moving boundary, $r=S(t)$, reaches a fixed boundary such as impermeable wool or the centre of the grain, $r=0$. 


\section{Transformed equations}

The governing equations (1-3) are transformed using

$$
z(r, t)=\frac{r-S(t)}{R(t)-S(t)},
$$

so that the region between the two moving boundaries is fixed as $z \in[0,1]$. Hence writing $u(r, t)=U(z, t)$ gives

$$
\begin{aligned}
\frac{\partial U}{\partial t}= & \frac{1}{(R-S)^{2}}\left(D(U) \frac{\partial^{2} U}{\partial z^{2}}+D^{\prime}(U)\left(\frac{\partial U}{\partial z}\right)^{2}\right. \\
& \left.+\alpha D(U) \frac{\partial U}{\partial z} \frac{(R-S)}{(z(R-S)+S)}\right)+\left(\frac{S^{\prime}+\left(R^{\prime}-S^{\prime}\right) z}{R-S}\right) \frac{\partial U}{\partial z}, \\
\frac{d R}{d t}= & \left.\frac{a}{R-S} \frac{\partial U}{\partial z}\right|_{z=1}, \quad \frac{d S}{d t}=\left.\frac{-b}{R-S} \frac{\partial U}{\partial z}\right|_{z=0},
\end{aligned}
$$

with $U(z=0, t)=0, U(z=1, t)=1, R(0)=S(0)=\alpha$. These equations are in cylindrical coordinates if $\alpha=1$ and Cartesian if $\alpha=0$.

Barry \& Caunce [2] showed that in the Cartesian situation exact solutions exist where $u(z(t), t)=U(z(t))$ only. The difficulty lies in finding $U(z)$ which is governed by the equation

$$
\frac{d}{d z}\left(D(U) \frac{d U}{d z}\right)=-a U_{z}(1)((1+\gamma) z-\gamma) \frac{d U}{d z},
$$

with $U(0)=0, U(1)=1$ and $\gamma=b U_{z}(0) /\left[a U_{z}(1)\right]$. This is a complicated nonlinear equation to solve, due not only to the diffusivity nonlinearity $D(U)$, but also because of the terms $U_{z}(0)$ and $U_{z}(1)$ which are unknowns. With this symmetry the boundary positions exhibit the classic $\sqrt{t}$ dependence:

$$
R=c_{r} \sqrt{t}, \quad \text { where } \quad c_{r}=\sqrt{\frac{2 a U_{z}(1)}{1+\gamma}},
$$




$$
S=-c_{s} \sqrt{t}, \quad \text { where } \quad c_{s}=\sqrt{\frac{2 b \gamma U_{z}(0)}{1+\gamma}}=\gamma c_{r},
$$

where $c_{r}$ and $c_{s}$ are constants. To find $U_{z}(1)$ and $U_{z}(0)$ is difficult and numerical methods are usually needed. However, some semi-analytic implicit solutions [2] exist for some restrictive cases, such as $D=1, a=b$, where

$$
U=\frac{\int_{0}^{z} \exp \left[-a U_{z}(0)\left(w^{2}-w\right)\right] d w}{\int_{0}^{1} \exp \left[-a U_{z}(0)\left(w^{2}-w\right)\right] d w},
$$

and

$$
U_{z}(0)=\frac{1}{\int_{0}^{1} \exp \left[-a U_{z}(0)\left(w^{2}-w\right)\right] d w}
$$

is an implicit equation with relatively simple numerical solution.

\section{$3 \quad$ Numerical Method}

Equation (6) is solved using central second order, finite differences for the spatial derivatives and a first order, forward difference for the time derivative (an Euler time-step). Hence equation (6) is written as

$$
U\left(z_{i}, t+d t\right)=U\left(z_{i}, t\right)+d t f\left[U\left(z_{i-1}, t\right), U\left(z_{i}, t\right), U\left(z_{i+1}, t\right), S(t), R(t)\right],
$$

where $z_{i}$ are the $N$ discretised spatial points for $i=1, \ldots, N ; d t$ is the time step; and $f$ the complicated discretised right-hand side of equation (6). Similar expressions are derived for equations (7). The time step, $d t$, is continuously modified with the maximum possible $d t$ chosen so that the solution remains stable. For example, in the initial stages when the boundary is moving rapidly, the time step is kept small, then increased as the boundary velocity slows down. In general the Cartesian situation is more stable as the cylindrical model has an additional advection-like term which can cause a 
numerically induced mass loss through the boundary if the time step is too large.

The difficulty in these schemes is finding the initial condition, since at time $t=0$ there is no region in which to solve and equation (6) has singularities where $R-S=0$. In the Cartesian case the routine is begun with $R-S=\epsilon \ll 1$, and $U(z, t)=z$. This approximation is stepped forward in time until $U(z, t) \rightarrow U(z)$. This solution can be checked by re-running the code from $R-S=\epsilon$ since $U(z)$ should remain unchanged over time. This procedure can also be checked against analytic solutions [2]. When $S(t)$ reaches the boundary at $q$ the boundary condition is changed to $\partial U / \partial z=0$ at $S(t)=q$ and the Euler time step continued until saturation.

The cylindrical case requires additional steps since no $U(z, t) \rightarrow U(z)$ solution exists. However, in the limit as $t \rightarrow 0$ the cylindrical governing equations reduce to the Cartesian equations since the cylindrical term

$$
\alpha D(U) \frac{\partial U}{\partial z} \frac{(R-S)}{(z(R-S)+S)}
$$

in equation (6) becomes zero if $R-S \rightarrow 0$. This is expected since in this limit a thin cylindrical shell appears as locally planar. Hence the initial condition for the cylindrical model is the $U(z)$ solution from the Cartesian model.

Even though the initial condition $U(z, t \rightarrow 0)$ has been found, the solution still needs a region in which to start. Initially the left boundary is set as $S(t \approx 0)=1-\epsilon$. The position of $R(t \approx 0)$ is then defined by conservation of solid mass, $1-\phi$. The solid mass is calculated as unswollen mass $m_{u}$, swollen mass $m_{s}$, and initial mass $m_{0}$, where $m_{u}+m_{s}=m_{0}$ or as integrals

$$
\int_{q}^{S(t)}\left(1-\phi_{i}\right) 2 \pi r d r+\int_{S(t)}^{R(t)}(1-\phi(r, t)) 2 \pi r d r=\int_{q}^{R(0)}\left(1-\phi_{i}\right) 2 \pi r d r .
$$

The initial radius of the grease is the known $R(0)$. The swollen mass can 
then be written in terms of nondimensional variables as

$$
\begin{aligned}
m_{s}= & \int_{S(t)}^{R(t)}(1-\phi(t)) 2 \pi r d r \\
= & 2 \pi \int_{0}^{1}\left(1-U(z, t)\left(\phi_{1}-\phi_{0}\right)+\phi_{0}\right)(z(R-S)+S) d z(R-S) \\
= & \pi\left(1-\phi_{0}\right)\left(R^{2}-S^{2}\right) \\
& -2 \pi\left(\phi_{1}-\phi_{0}\right)\left[(R-S)^{2} \int_{0}^{1} z U(z, t) d z+(R-S) S \int_{0}^{1} U(z, t) d z\right]
\end{aligned}
$$

Re-arrange this to find

$$
R(t)=\frac{1}{\phi_{a}}\left(S\left(\phi_{a}-\phi_{b}\right)-\sqrt{\left(S \phi_{b}\right)^{2}+\frac{m_{s} \phi_{a}}{\pi}}\right),
$$

where

$$
\begin{aligned}
& \phi_{a}=\left(1-\phi_{0}\right)-2\left(\phi_{1}-\phi_{0}\right) \int_{0}^{1} z U(z, t) d z, \\
& \phi_{b}=\left(1-\phi_{0}\right)-\left(\phi_{1}-\phi_{0}\right) \int_{0}^{1} U(z, t) d z,
\end{aligned}
$$

are convenient shorthands. Writing $m_{s}=m_{0}-m_{u}$ gives

$$
m_{s}=\pi\left(1-\phi_{i}\right)\left(R(0)^{2}-S^{2}\right),
$$

noting that by scaling $R(0)=1$, which is then substituted into equation (17).

Hence substituting $U(z, 0)=U(z)$ and $S=1-\epsilon$ gives a required estimate for the initial position of $R$. This same procedure can be done for the Cartesian case, although since $U(z, t) \rightarrow U(z)$ then $R(t)$ and $S(t)$ can be found, using equations (9) and (10), up to when $S(t) \rightarrow q$. 
This same mass balance can be used to find the final swelling radius by substituting $U(z, \infty)=1$ and $S=q$ to obtain

$$
R(\infty)=\sqrt{\frac{1-\phi_{i}}{1-\phi_{1}}-q^{2} \frac{\phi_{i}-\phi_{1}}{1-\phi_{1}}} .
$$

An important and practical outcome of this analysis is the time for the material to swell. Numerically this is defined as when $u(S(t), t)=0.99$, that is when the inner-most region has swollen to within one percent of its final value.

A scaling argument can also be used to estimate the swelling time where the typical time scale is

$$
t_{s}=\frac{(R(\infty)-q)^{2}}{D(1)}=(R(\infty)-q)^{2},
$$

where scaling implies $D(1)=1$. This is the 'typical non-dimensional' time for the material to swell completely.

\section{Results}

All the results shown are calculated with typical values of $\phi_{i}=0$ initially, $\phi_{0}=0.6$ when the grease begins to swell at $S$ and $\phi_{1}=0.8$ when the grease is fully swollen. The dimensional diffusion coefficient

$$
D^{*}(\phi)=e^{c \phi}, \quad \rightarrow \quad D(u)=e^{\nu(u-1)},
$$

where $c=5.22[7]$ and hence $\nu=c\left(\phi_{1}-\phi_{0}\right)=10.44$.

Figure 2 shows the boundary positions as a function of time for two different wool widths $q=0.5$ and $q=0.05$. The $S(t)$ boundary can be 
seen to progress to the $q$ limit and then stop. The $R(t)$ boundary progresses uniformly to the maximum value calculated in equation (21). Also worth noting is that $S(t)$ accelerates near $r=0$ due to the diminished volumes for small $r$ (that is $2 \pi r d r$ decreases). Hence the volume of fluid diffusing through the medium pushes the $S(t)$ boundary further and faster. Once the boundary $q$ is reached the boundary at $R(t)$ slows down. This is not intuitively obvious but occurs because the motion of the $S(t)$ boundary helps drive the flow.

In Figure 3 the moisture fraction $U(z, t)$ is shown as a function of $z$ for different times. In the Cartesian case the curves for small time, those bounded by $U(0, t)=0$ and $U(1, t)=1$, would be the one curve $U(z, t)=$ $U(z)$ identical to the $t=0$ curve shown in Figure 3. In this cylindrical case $U(z, t)$ slowly changes for the early times, and when the boundary at $q$ is reached the boundary condition changes and the moisture fraction increases to eventual saturation.

Figure 4 shows the grease swelling time as a function the nondimensionalised thickness of the wool fibre, $q$. Equation (22) gives the approximation for the time to fully swell the grease. The partial swelling time is numerically found when $S(t)$ reaches $q$. We note that by symmetry, rotation of the $S(t)$ curve in Figure 2 gives this partial swelling time as a function of $q$. The full swelling time is calculated numerically by finding when $U(s(t), t)=0.99$. The $x$-axis of this figure corresponds to the proportion of grease, hence when $1-q=1$ then no wool fibre exists and the cylinder is all grease. This is physically equivalent to the wheat and rice swelling cases. When $1-q=0$ there is little material to swell, hence swelling time is zero. Note that plotted is non-dimensional time, which has been scaled with respect to initial wool and grease radius. 


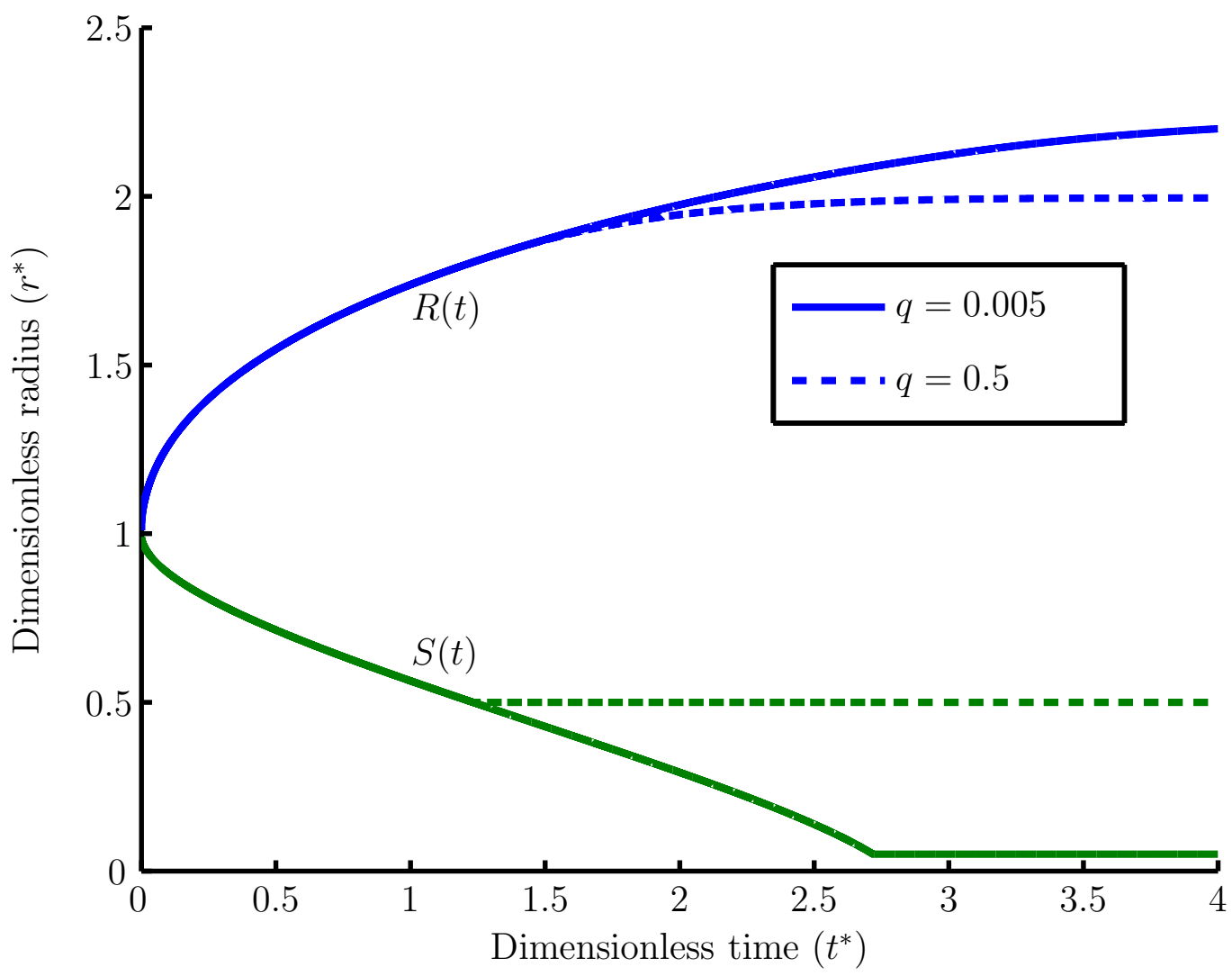

Figure 2: Boundary positions versus time for two non-dimensional wool widths, $q=0.5$ and $q=0.05$. 


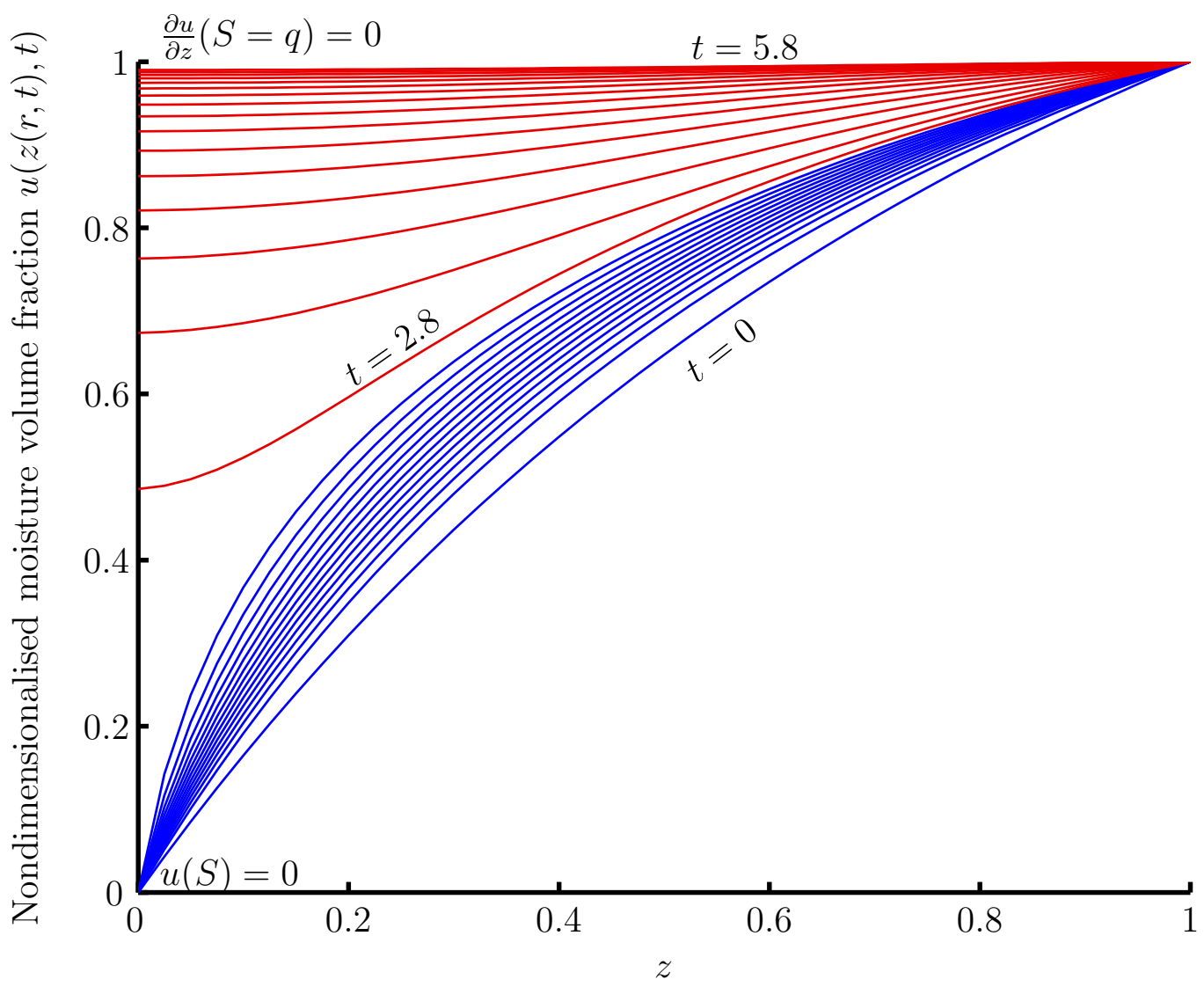

Figure 3: Moisture fraction $U(z, t)$ as a function of $z$ for different times. Note the change in behaviour when the boundary $S(t)$ reaches $S=q$ and the boundary condition changes from $u=0$ to $\partial u / \partial z=0$. 


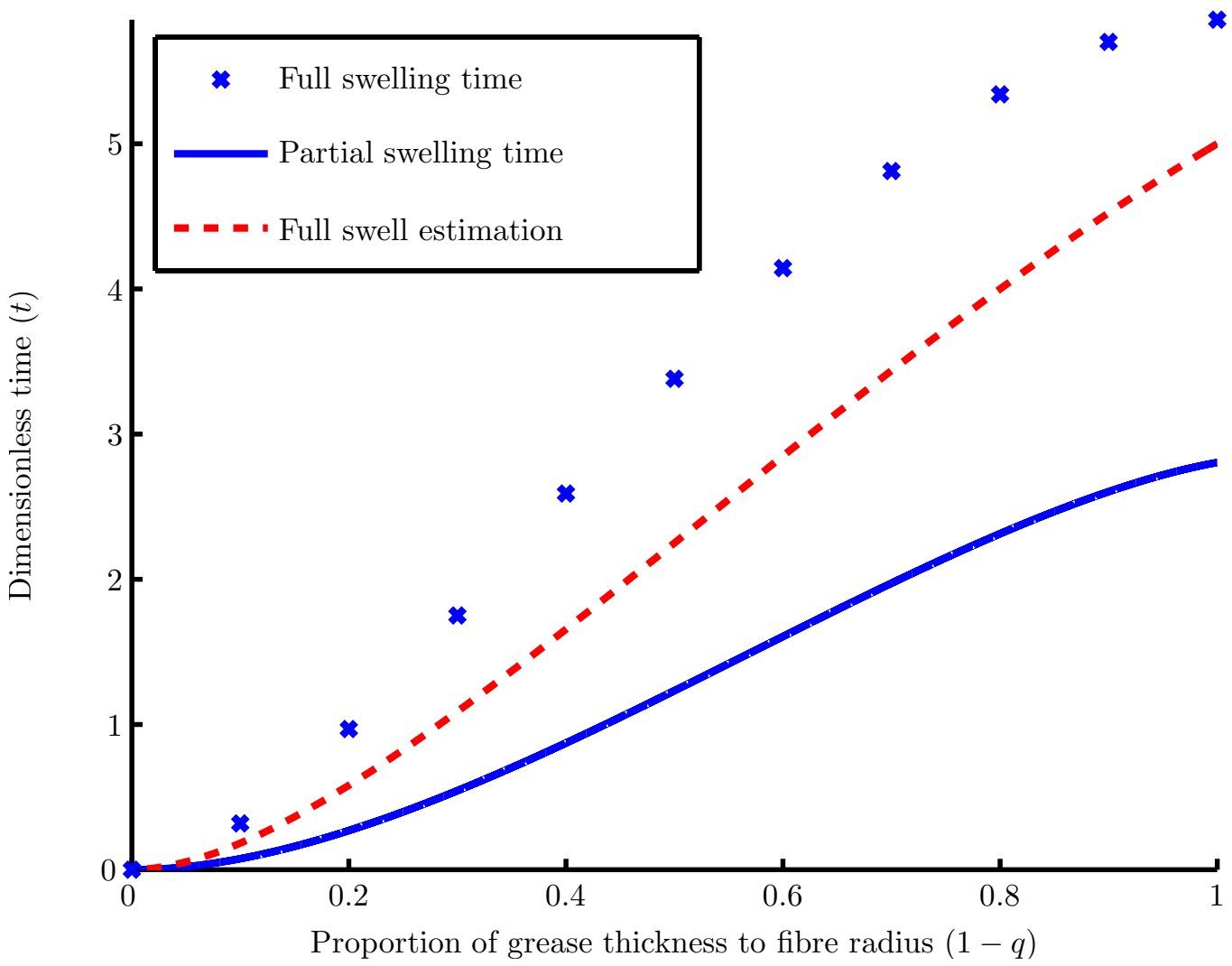

Figure 4: Time for a material to swell versus scaled width of grease. Equation (22) gives the estimated time. 


\section{Conclusion}

We developed numerical and analytic methods to analyse diffusion between two moving boundaries, a model of swelling grease on a wool fibre. The numerical solution involves applying a boundary-fixing transformation and using finite differences. The difficulty lies in finding the initial condition with which to begin the numerical iteration. We found that the Cartesian solution is invariant in time, hence allowing easy calculation, and this solution was the initial condition for the cylindrical problem. These solutions allowed estimates of the swelling time to be made as a function of grease depth.

\section{References}

[1] Anderson, C. A., A new picture of the raw-wool fibre. J. Text. Inst., 1982, 6, 289-292. C122

[2] Barry, S. I, and Caunce, J, Exact and numerical solutions to a Stefan problem with two moving boundaries. Applied Mathematical Modelling, (accepted 2006). C122, C123, C124, C125

[3] Barry, S. I, Marchant, T. and Mercer, G. N., Grease recovery and dirt removal in wool scouring. Mathematics in Industry Study Group, Proceedings, ed: J. Hewitt and K. White, 2002. C122

[4] Caunce, J., Barry, S. I., Mercer, G. N. and Marchant, T., Modelling a wool scour bowl. ANZIAM Journal E, 47, C19-C33, 2006. http://anziamj . austms.org.au/V47EMAC2005/Caunce3 C122

[5] Caunce, J., Barry, S. I., and Mercer, G. N., A simple mathematical model of wool scouring ANZIAM Journal E, 47, C34-C47, 2006. http://anziamj.austms.org.au/V47EMAC2005/Caunce2 C122 
[6] Crank, J., Free and moving boundary value problems, Oxford University Press, Oxford, UK, 1984. C120, C122

[7] Davey, M., Landman, K., McGuiness, M. and Jin, H. , Mathematical modelling of rice cooking and dissolution in beer production. AIChE Journal, 2002, 48 (8), 1811-1826. doi:10.1002/aic.690480821 C120, $\mathrm{C} 122, \mathrm{C} 127$

[8] Hill, J. M., One-dimensional Stefan problems: An introduction. Longman Scientific and Technical, New York, USA, 1987. C120, C122

[9] McGuiness, M., Please, C., Fowkes, N., McGowan, P., Ryder, L., and Forte, D. Modelling the wetting and cooking of a single cereal grain. IMA J. of Math. Appl. Bus. Ind. 2000, 11, 49-70. doi:10.1093/imaman/11.1.49 C120, C122 


\section{Author addresses}

1. J. F. Caunce, School Physical, Environmental \& Mathematical Sciences, University of New South Wales @ ADFA, Canberra, Australia.

2. S. I. Barry, School Physical, Environmental \& Mathematical Sciences, University of New South Wales @ ADFA, Canberra, Australia.

mailto:s.barry@adfa.edu.au 